\title{
Verbal Fluency, Speech-Language and Neurocognitive Outcomes in Youth with Cochlear Implants
}

\author{
Fahad Hasnain ${ }^{1}$, David Pisoni ${ }^{1,2}$, William Kronenberger ${ }^{1,2}$
}

${ }^{1}$ Indiana University School of Medicine, DeVault Otologic Research Laboratory, Department of Otolaryngology-Head \& Neck Surgery, ${ }^{2}$ Indiana University School of Medicine, Department of

Psychiatry

Background: Cochlear Implants (Cls) restore some hearing to deaf children, although some language domains such as verbal fluency (VF) are at risk for delay. VF, the ability to efficiently retrieve words from the mental lexicon, is critically important for learning, reasoning, and memory. In this study we used a novel assessment paradigm to understand how VF develops in youth with $\mathrm{Cls}$ and to investigate associations between VF, speech-language, and neurocognitive functioning. We hypothesized that phonemic VF (retrieving words based on sounds) would show greater delays than semantic VF (retrieving words within category) and that $\mathrm{VF}$ would relate to spoken language and memory outcomes.

Methods: 28 prelingually deaf, early-implanted ( $<4$ years), long-term ( $\geq 7$ years) child and adolescent users of Cls were compared with 33 age and nonverbal IQ-matched normal-hearing $(\mathrm{NH})$ peers. VF measures were compared between $\mathrm{Cl}$ and $\mathrm{NH}$ and were correlated with speech, language, and neurocognitive outcomes.

Results: Compared to $\mathrm{NH}$ peers, youth with Cls retrieved fewer words, had longer start latencies, and fewer word clusters in the phonemic VF test. Stronger phonemic VF in the $\mathrm{Cl}$ sample was associated with better speech perception and language. Stronger VF in both samples was associated with better short-term/working memory and inhibition/concentration.

Conclusion: VF based on phonological (sound) characteristics of words is delayed in youth with Cls, whereas VF based on semantic meaning is relatively spared. Phonemic VF delays have downstream effects on speech perception and language in youth with Cls, whereas both types of VF are associated with verbal short-term/working memory and executive functioning.

Clinical Policy Impact and Implications: Study results indicate a need for assessment and intervention targeting VF (especially phonemic) in youth with Cls as a potential method for improving speech perception, language, verbal memory, and inhibition-concentration outcomes. 